\title{
The Effects of Humanized Psychological Nursing Model in General Surgery Nursing
}

\author{
Guifeng Xue ${ }^{1 *}$, Huafang Yi¹, Ping Xue ${ }^{1}$, Wenmin Sun ${ }^{2}$ \\ ${ }^{1}$ The Second People's Hospital of Taizhou City, Jiangsu Province, Taizhou 225500, Jiangsu Province, China \\ 2The First People's Hospital of Yancheng City, Yancheng 224000, Jiangsu Province, China
}

*Corresponding author: Guifeng Xue, 13775796086@163.com

\begin{abstract}
Objective: To analyze and comprehensively study the clinical effects of humanized psychological nursing model in general surgery nursing. Methods: The study period was from January 2018 to December 2020. A sample of 200 patients who were admitted to The Second People's Hospital of Taizhou City for general surgery were selected. Random lottery grouping was used to divide the subjects into a study group and a control group. The sample within each group was $n=100$. The patients in the control group were provided with conventional general surgery nursing plan whereas the patients in the study group received the same nursing plan but with addition of the humanized psychological nursing model. The indicators of the two groups were compared and analyzed. Results: Comparing the scores from Hamilton Anxiety Rating Scale (HAMA) and Hamilton Depression Rating Scale (HAMD) after nursing intervention, postoperative visual analog scale (VAS), hospital stay, and patient satisfaction with the nursing services between the two groups, the study group was better $(\mathrm{P}<0.05)$. Conclusion: The implementation of humanized psychological nursing model in general surgery nursing had a significant effect in which there were improvements in regard to the patients' mental state and their satisfaction with the nursing services. Hence, it is worthy of promotion.
\end{abstract}

Keywords: General surgery; Humanized psychological nursing; Effect

Publication date: July 2021; Online publication: July 30, 2021

\section{Introduction}

General surgery is an important division of medical institutions at all levels. They have large numbers of patients with complex and diverse diseases in which most of them require surgery. In the treatment of these patients that are affected by factors such as their illnesses and the surgeries, they often have negative emotional states which lead to poor treatment compliance and prognosis. In order to improve the treatment effect among these patients, effective nursing intervention measures need to be taken ${ }^{[1]}$. Humanized psychological care is a new nursing model whereby improving patients' comfort is the main nursing goal. Incorporating humanized care into routine physiological care would help improve the psychological state of patients and increase their satisfaction with nursing services ${ }^{[2]}$. In order to deeply explore and analyze the application value of humanized psychological nursing in general surgery, this research summarized, analyzed, and comprehensively studied the basic data of general surgery patients in The Second People's Hospital of Taizhou City and the related problems in the implementation of humanized psychological nursing. 


\section{Materials and methods}

\subsection{General information}

The study period was from January 2018 to December 2020. A sample of 200 patients that were admitted to The Second People's Hospital of Taizhou City for general surgery were selected and the random lottery grouping method was used to divide the patients into a study group and a control group. The sample within each group was $n=100$. All the patients had diagnosis pertaining to general surgery diseases, without cognitive impairment, and informed consent was taken. The basic data of the two groups were summarized and analyzed. The study group had 52 male patients and 48 female patients, age ranging from 22 to 68 years old with an average age of $45.59 \pm 2.78$ years old. Among them, there were 12 cases of acute cholecystitis, 10 cases of appendicitis, 15 cases of gallstones, 13 cases of breast tumor, 18 cases of gastric cancer, 10 cases of rectal cancer, 14 cases of colon cancer, and 8 cases of thyroid adenoma. On the other hand, there were 51 male patients and 49 female patients in the control group, age ranging from 24 to 67 years old with an average age of $45.65 \pm 2.84$ years old. Among them, there were 14 cases of acute cholecystitis, 12 cases of appendicitis, 14 cases of gallstones, 10 cases of breast tumors, 17 cases of gastric cancer, 9 cases of rectal cancer, 14 cases of colon cancer, and 10 cases of thyroid adenoma. The differences in the baseline data had no effect on the conclusion of this study $(\mathrm{P}>0.05)$.

\subsection{Method}

Patients in the control group received routine surgical care. Nursing staffs assisted patients in various general surgical examinations, explained disease knowledge, instructed patients in the consumption of daily medications, assisted surgeons during surgery, and provided basic post-surgical rehabilitation nursing interventions.

The patients in the study group received the same nursing care as the control group with the addition of humanized psychological nursing. The nursing staff analyzed the patients' treatment process, summarized their psychological characteristics, determined the patients' nursing service needs, and adopted targeted humanized psychological nursing intervention measures. (1) Initial care of admission. General surgery patients would feel unfamiliar with other patients in the hospital during admission and would be in severe emotional states. Based on the humanized psychological nursing intervention, nursing staffs were required to actively and warmly welcome these newly admitted patients, guide them to familiarize themselves with the hospital's environment, introduce the medical staffs, explain relevant systems in the hospital, and answer their questions in order to ease their nervousness and fearful emotional states. (2) Preoperative visit. For patients undergoing surgical treatment, the nursing staffs were required to carry out preoperative visits. During these visits, in addition to assisting the doctors in the completion of preoperative assessments, nursing staffs were required to explain the surgical procedures, pre-operative fasting and water prohibition, surgical cooperation, and other precautions which emphasized on the efficacy and safety of the surgery to the patients in order to relieve their nervousness and improve treatment compliance. (3) Intraoperative care. Before pushing the patients into the operating theater, the nursing staffs were required to adjust the temperature and humidity of the room as well as properly and accurately prepare all the necessary surgical drugs and instruments. After the patients were pushed in, they were required to adjust their positions while the nursing staffs had to inform them to maintain stable moods, keep them warm, and cover their private parts so that they would feel respected and understood. (4) Postoperative humanized nursing. In order to shorten the postoperative recovery time of these patients, postoperative nursing staffs were required to perform standardized catheter care and condition monitoring. The patients would then be informed of the success of the surgery after they had woken up from the wearing effect of the anesthesia. During the postoperative nursing intervention period, nursing staffs were required to provide oral care, skin 
care, catheter care, and comprehensive patient-centered medical care services to actively prevent various common complications after surgery, improve patients' physical and mental health, as well as promote recovery from their illnesses. At the same time, the nursing staffs were required to provide psychological counseling throughout the whole process, answer in detail the various questions raised by the patients in regard to their diseases, provide positive encouragement and guidance, organize communication activities between patients, and inform the patients' family members of the need to accompany the patients more so that the patients would be able to recover during the postoperative period and for the maintenance of good mental health.

\subsection{Evaluation}

The scores from HAMA (Hamilton Anxiety Rating Scale) and HAMD (Hamilton Depression Rating Scale) before and after nursing intervention were compared between the two groups. The scores were directly proportional to the degree of anxiety and depression.

The postoperative VAS, hospital stay, and patient satisfaction with the nursing services were also compared between the two groups.

\subsection{Statistical analysis}

Statistical Package for the Social Sciences (SPSS) version 23.0 software was used to analyze various data. In this study, the measurement data was $( \pm s)$, the count data was $(\%)$, and the test methods were t-test and chi-square $\left(\mathrm{X}^{2}\right) . \mathrm{P}<0.05$ indicated differences between the groups.

\section{Results}

\subsection{Comparison of the scores from HAMA and HAMD before and after nursing intervention between the two groups}

Comparing the scores from HAMA and HAMD before nursing intervention between the two groups, there was no significant difference $(\mathrm{P}>0.05)$. In regard to that, the study group was better after the nursing intervention $(\mathrm{P}<0.05)$.

Table 1. Comparison of scores from HAMA and HAMD before and after nursing intervention between the two groups $( \pm \mathrm{s})$

\begin{tabular}{ccccc}
\hline Group & \multicolumn{2}{c}{ Before care } & \multicolumn{2}{c}{ After care } \\
\cline { 2 - 5 } & HAMA & HAMD & HAMA & HAMD \\
\hline Study group $(\mathrm{n}=100)$ & $49.27 \pm 2.26$ & $49.15 \pm 2.33$ & $41.16 \pm 1.04$ & $41.38 \pm 1.14$ \\
Control group $(\mathrm{n}=100)$ & $49.35 \pm 2.24$ & $49.06 \pm 2.48$ & $45.88 \pm 1.74$ & $44.36 \pm 1.28$ \\
$\mathrm{t}$ value & 0.251 & 0.264 & 23.284 & 17.385 \\
P value & 0.801 & 0.791 & 0.000 & 0.000 \\
\hline
\end{tabular}

\subsection{Comparison of postoperative VAS, hospital stay, and patient satisfaction with the nursing services between the two groups}

Comparing the postoperative VAS, hospital stay, and patient satisfaction with the nursing services between the two groups, the study group was better after the nursing intervention $(\mathrm{P}<0.05)$. 
Table 2. Comparison of postoperative VAS, hospital stay, and patient satisfaction between the two groups

\begin{tabular}{cccc}
\hline Group & Postoperative VAS & Hospital stay & Patient satisfaction \\
\hline Study group $(\mathrm{n}=100)$ & $1.28 \pm 0.35$ & $4.83 \pm 1.25$ & $98(98.0 \%)$ \\
Control group $(\mathrm{n}=100)$ & $2.73 \pm 0.56$ & $6.92 \pm 1.47$ & $82(82.0 \%)$ \\
$\mathrm{X}^{2} / \mathrm{t}$ value & 21.957 & 10.831 & 14.222 \\
P value & 0.000 & 0.000 & 0.000 \\
\hline
\end{tabular}

\section{Discussion}

Common types of diseases in general surgery include appendicitis, cholecystitis, gallstones, etc. Most patients require surgical intervention. Patients with general surgery diseases are affected by their conditions and other factors during the treatment. They often have negative psychological states such as anxiety, depression, and fearfulness. Their degree of compliance to treatment is poor and their bodies' stress response is serious ${ }^{[3]}$. In order to improve the treatment effect of general surgical diseases and alter the mental state of patients, effective nursing intervention measures are needed.

Humanized psychological nursing model is a nursing program widely used in general surgery clinics in recent years. Compared with the conventional nursing model, its core concept is patient-centered in addition to various nursing care that is refined according to the condition of the patients and their basic needs of nursing services. Intervention measures can significantly improve nursing plans and patient satisfaction ${ }^{[4]}$. With the humanized psychological nursing intervention in general surgery, the nursing staffs are able to replace the conventional passive nursing service model with an active one, ensuring a good service attitude, effectively integrating physiological with psychological nursing, as well as enabling patients to feel respected and understood. In this way, patients would then actively comply to various treatments and nursing intervention measures in addition to an improvement to the overall quality of nursing intervention and satisfaction of patients with nursing services ${ }^{[5]}$.

In this study, the nursing staffs had carried out comprehensive analysis and research on the condition of the surgical patients during their admission, introduced the basic course of their treatment, assessed their mental states, as well as implemented preliminary psychological care and health education which alleviated their unfamiliarity ${ }^{[6-7]}$. During preoperative visits, the nursing staffs had established a humanized service concept. In addition to informing the patients of surgical precautions, measures such as psychological comfort and patient introduction were provided which effectively improved patients' negative emotions before the surgery and relieved their bodies' stress response in addition to the improvement of their compliance. In post-operative nursing, nursing staffs had integrated psychological intervention throughout the whole process with intervention models such as rehabilitation nursing which can effectively alleviate postoperative pain and other discomforts, shorten the recovery time, and improve their overall satisfaction with the nursing services.

To summarize and analyze this study, the scores from HAMA and HAMD of the patients in the study group after nursing intervention were better than those in the control group, suggesting that humanized psychological care can improve the emotional states of patients such as anxiety and depression. The postoperative VAS, hospital stay, and patient satisfaction with the nursing services of the patients in the study group were also better than those in the control group, suggesting that humanized psychological care can relieve postoperative pain, shorten the hospital stay, and improve patient satisfaction with the nursing services. However, humanized psychological nursing requires relatively high level of comprehensive skills from the nursing staffs in which the hospitals would need to organize training and education for their nursing staffs on a regular basis and change the concept of nursing services to ensure the overall quality of humanized nursing service ${ }^{[8]}$. 
Based on the above analysis, it can be seen that the implementation of humanized psychological nursing model in general surgical nursing had a significant effect which improved the psychological state of patients and increased their satisfaction with the provided nursing services. Hence, it is worthy of promotion.

\section{Disclosure statement}

The authors declare that there is no conflict of interest.

\section{References}

[1] Li H, 2021, Analysis of the Application Effect of Comfort Nursing in the Nursing Management of Laparoscopic Surgery in General Surgery. Health Vision, (1): 278-9.

[2] Qian L, Sun J, Wang Y, et al., 2021, Study on the Applicability of Classification of Nursing Measures for Children's General Surgery Nursing Records. Chinese Journal of Practical Nursing, 37(2): 139-44.

[3] Xue H, 2021, The Effect of Nursing Training in General Surgery Clinical Nursing and Strategies to Cope with Unsafe Factors in Rehabilitation. Chinese Community Physician, 37(1): 151-2.

[4] Gu L, 2021, Discuss the Effect of Rapid Rehabilitation Nursing Application in General Surgery on the Rehabilitation Effect and Quality of Life of Patients. Special Health, (26): 214.

[5] Yuan X, 2021, The Clinical Effect of Continuous Quality Improvement of General Surgical Pipeline Nursing on Patients with Surgical Catheterization. Special Health, (3): 256.

[6] Lin X, 2021, The Effect of Pain Nursing Intervention on Reducing Postoperative Pain in General Surgery Patients. China Health Care and Nutrition, 31(6): 124.

[7] Han J, Han M, 2021, Analysis of Perioperative Nursing Points for Patients Undergoing General Surgery for Severe Pancreatitis. Health Must Read, (6): 101.

[8] Liu D, 2021, Perioperative Psychological Analysis and Nursing Countermeasures for Elderly Patients in General Surgery. Special Health, (27): 241. 